\title{
Oligometastases: history of a hypothesis
}

\author{
Michael T. Milano ${ }^{1}$, Tithi Biswas ${ }^{2}$, Charles B. Simone II $^{3}$, Simon S. Lo ${ }^{4}$ \\ ${ }^{1}$ Department of Radiation Oncology, University of Rochester, Rochester, NY, USA; ${ }^{2}$ Department of Radiation Oncology, University Hospitals \\ Seidman Cancer Center, Case Western Reserve University, Cleveland, OH, USA; ${ }^{3}$ Department of Radiation Oncology, Memorial Sloan Kettering \\ Cancer Center and New York Proton Center, New York, NY, USA; ${ }^{4}$ Department of Radiation Oncology, University of Washington School of \\ Medicine, Seattle, WA, USA \\ Contributions: (I) Conception and design: All authors; (II) Administrative support: None; (III) Provision of study materials or patients: None; (IV) \\ Collection and assembly of data: None; (V) Data analysis and interpretation: None; (VI) Manuscript writing: All authors; (VII) Final approval of \\ manuscript: All authors. \\ Correspondence to: Michael T. Milano. Department of Radiation Oncology, University of Rochester, 601 Elmwood Ave. Box 647, Rochester, NY \\ 14642, USA. Email: michael_milano@urmc.rochester.edu.
}

\begin{abstract}
The term oligometastases represents a clinical state of metastatic disease that is limited in the number of metastatic sites and extent of disease, and amenable to metastasis-directed surgical or ablative therapy. While metastasis-directed approaches are used for palliation, the primary goal of such treatment for patients with oligometastases is to prolong survival and the duration of cancer control. Metastasis-directed therapy, for patients with limited number of metastatic sites, has been practiced for decades, dating back to the era before chemotherapy was widely used. Systemic therapy has become the accepted standard of care for metastatic disease. And while not curative for most solid cancers, systemic therapy can delay cancer progression, prolong life, and maintain or improve quality of life, albeit often at the expense of toxicities which can adversely impact quality of life. From the 1960s to 1980 s, prominent physicians questioned whether metastasis-directed resection or radiotherapy could potentially be curative treatment approaches. In 1995, Drs. Hellman and Weichselbaum wrote an editorial that coined the term "oligometastases" and refined the hypothesis of metastasis-directed surgical and radiotherapeutic treatments as potentially curative for select patients. Their article was the first to explicitly describe the clinical state of metastases existing along a spectrum, with a spectrum of behaviors (ranging from indolent disease confined to limited sites to widespread disease) and, therefore, a spectrum of potential treatments. In the ensuing decades, there were rapid technologic advancements in radiotherapy, including stereotactic body radiation therapy (SBRT), which facilitated delivery of ablative doses of radiation to precisely and accurately targeted tumors. SBRT has been considered an optimal non-surgical approach to treat oligometastases, allowing for definitive-dose delivery and for targeting accuracy that minimizes normal tissue radiation exposure. In the early 2000s, many institutions began publishing prospective studies demonstrating favorable outcomes in patients with oligometastases treated with SBRT. Not answered in these single-arm studies was whether patients generally fared better than expected due to selection of patients with relatively indolent disease, or from metastasisdirected treatment. There is also a potential for immortal time bias with non-randomized comparisons. However, recent randomized phase II studies have suggested that SBRT for oligometastases is associated with improved survival outcomes. Phase III studies, many specific for certain cancers (i.e., breast, prostate or lung cancers) are accruing. Future work will be needed to identify which patients are most apt to benefit from metastasis-directed therapy; in addition to clinical factors, host and/or tumor genomics may prove to be prognostic. Metastasis-directed therapy may become more important with improvements in systemic therapy in controlling micrometastatic disease. Incorporating immunotherapy with SBRT may also be a promising approach, with SBRT perhaps augmenting the immune response. As personalized medicine evolves, patients with oligometastases will be better served. The history of oligometastases will continue to unfold.
\end{abstract}

Keywords: Metastases; oligometastases; stereotactic body radiation therapy; local therapy; prognosis 
Submitted Feb 22, 2020. Accepted for publication Mar 09, 2020.

doi: 10.21037/apm.2020.03.31

View this article at: http://dx.doi.org/10.21037/apm.2020.03.31

\section{Metastatic disease}

Metastatic disease implies the spread of cancer to sites removed from the primary cancer site, and subsequent growth at those sites outside of the primary cancer. In order for distant metastatic spread to occur, cancer cells must separate from the primary cancer, enter the blood stream (intravasation), exit the blood stream (extravasation) and then colonize and grow in a new environment (1). As part of this process, the cancer cells may undergo several genotypic and phenotypic changes. Metastatic disease can be clinically apparent or occult (i.e., not readily seen by clinical examination or radiography). Conceptually, the presence of circulating tumor cells in the blood (which are not routinely accessed for staging or other purposes) in the absence of clinically apparent metastases is a manifestation of occult metastatic disease. Metastases too small to be detectable on imaging, but with the propensity to grow, would also be considered occult. This represents a clinically meaningful scenario, as these metastases have seeded elsewhere and have begun to grow. It is the propensity for metastases to spread and then grow at distant sites that make metastatic disease life-threatening. For some locally advanced cancers, or early-stage cancers with high risk of occult metastatic disease (such as small cell cancers or primitive neuroectodermal tumors), it is assumed that a patient, without radiographic evidence of metastases, has a high risk of occult metastatic disease at the time of initial presentation. Cancer is staged as metastatic once the metastatic disease is clinically apparent. Generally, this is considered an incurable state of disease.

\section{Treatment of metastatic disease}

Since the mid-1900s, the primary treatment for metastatic disease has been systemic chemotherapy, with the goal of controlling the cancer for a duration of time and prolonging life. The potential benefit in quality of life (by slowing cancer progression) from cytotoxic chemotherapy is balanced against the risks of toxicities. Recent developments in biologically targeted therapies and immunotherapy have led to impressive improvements in patient outcomes. While such drugs have potentially debilitating side effects, they are often much better tolerated than cytotoxic chemotherapy. Notably, outside of hematologic malignancies and germ cell tumors, systemic therapy-whether chemotherapy, biologically targeted therapy or immunotherapy-is rarely considered a curative treatment.

For non-metastatic disease, surgery, radiotherapy or other ablative treatments, often combined with systemic therapy for more advanced stages of disease, are the mainstay of curative therapies for most solid cancers. For patients amenable to treatment with definitive cancerdirected therapy (such as resection and/or radiotherapy), but for whom there is a high risk of harboring occult metastatic disease, systemic therapy can be used neoadjuvantly (before cancer-directed therapy), concurrently with radiotherapy and/or adjuvantly (after cancer-directed therapy).

For metastatic disease, local treatment modalities (meaning the treatment is targeted to a specific cancer site or region) are commonly used to palliate symptoms from mass effect and/or invasion of cancer; such symptoms and manifestations might include pain, airway obstruction, bleeding, erosion through skin or mucosa, or neurologic deficits from intracranial or spinal axis metastases causing compression. However, one could envision scenarios in which such treatments could be considered a potentially curative option for patients whose metastatic disease appears to be confined to a limited number of sites, with a relatively low burden of metastatic disease. This clinical state is often termed "oligometastatic" with "oligo" meaning "few". For those patients with oligometastases, it may be reasonable to be more aggressive with localized treatment modalities in conjunction with systemic therapy; in other words, the metastasis-directed therapies would be similar in intent to those treatments being used for the definitive treatment of non-metastatic cancer. For radiotherapy, this would imply using higher doses than what are typically used for palliation, in an effort to provide more durable control of the treated metastases, as well as potentially eradicating all sites of metastatic disease.

\section{Oligometastatic disease-brief history}

While there has been much excitement recently about the treatment of oligometastatic disease with radiotherapy or 
surgery, the use of these treatments for limited metastatic disease dates back decades (2-4). This is largely attributable to the fact that radiotherapy (first used in the mid-1890s) and surgery were the only established oncologic therapies for patients treated in the era before hormonal therapy and chemotherapy. Over 50 years ago, Phillip Rubin and Jerold Green wrote a book entitled "Solitary Metastases" in which they summarized decades of data and explored the concept of possible curative-intent treatments in patients with one metastasis seen on $\mathrm{X}$-ray imaging (computerized tomography had not yet been invented) (2). That same year (in 1968), Rubin published an editorial in which he questioned "Are metastases curable?" (5). In addition to focusing on solitary metastases, he described "delayed metastases" or "metachronous metastases" that develop some time after treatment of the primary cancer and "synchronous metastases" that are diagnosed at the same time as the primary cancer. The terminology of metachronous and synchronous metastases is still used today (as described below), with the former being thought to represent more relatively indolent disease. In Rubin's 1968 FAMA editorial, he also contemplated "immunologic considerations" and cancer cell antigens as potentially important factors for curing metastatic disease, perhaps showing great foresight into the types of treatments that would develop over the ensuing decades.

In 1984, Lester Peters, Luka Milas and Gilbert Fletcher wrote a paper entitled "The role of radiation therapy in the curative treatment of metastatic disease" that stemmed from a 1983 "Symposium of Fundaments Cancer Research" (4). They described that tumor size was an important factor for detectability on imaging (notably before the era of magnetic resonance imaging and positron emission tomography), as well as the radiotherapy doses needed to "cure" a given sized tumor. This paper was written in the era before computerized tomography (CT) imaging was incorporated into radiotherapy planning - a technology not becoming widely used until the 1990s. This technology allows for radiotherapy to be more conformally delivered to treatment targets. In the 1980s, diagnostic CT imaging was used to discern where tumors were located, and the treatment fields (encompassing tumor and subclinical nodal sites) were designed based upon bony anatomy and/or tumors visible on plain radiographs. With this technology, Peters, Milas and Fletcher expressed concern about delivering therapeutic doses to metastases "without producing unacceptable normal tissue injury."

In 1995, Samuel Hellman and Ralph Weichselbaum wrote an editorial in $7 \mathrm{Clin}$ Oncol, that for the first time, explicitly defined oligometastases and how patients with oligometastatic disease might be amenable to curativeintent metastasis-directed treatment. Notably, Hellman's 1994 Karnofsky lecture preceded, and to a large extent laid the groundwork for, the classic 1995 Hellman and Weichselbaum editorial. In his Karnofsky lecture, Hellman focused on "small breast cancers" and introduced the concept that cancer is "a heterogeneous disease (with) a spectrum of proclivities from a disease that remains local throughout its course to one that is systemic when first detectable" (6). His "spectrum" hypothesis was distinct from the model of William Halsted (the renowned surgeon from Johns Hopkins Hospital) in which he postulated that breast cancer progresses in an orderly manner from the primary site, and then nodal sites and then to distant metastatic sites. This orderly progression formed the basis for oncologic resection of non-metastatic breast cancer.

At the other extreme is the notion that breast cancer is a systemic disease from inception, even in patients with small tumors; Bernard Fisher is often credited with this model, though Hellman noted that Geoffrey Keynes and George Crile, Jr. had initially postulated this hypothesis before Fisher. Hellman's spectrum model represented an intermediate theory between the Halstedian model and that of Keynes, Crile and Fisher. While this spectrum model was initially framed in the setting of small breast cancers-the notion of cancer existing along a spectrum was readily extrapolated to metastatic disease. Hellman and Weichselbaum's classic editorial explicitly proposed "the existence of a clinical significant state of oligometastases" as a disease state along a "spectrum of malignancy" (7). Oligometastases were described as "limited in number and location" for which "the facility for metastatic growth has not been fully developed...”. Importantly, they also claimed that, "Not only is there a spectrum of malignancy... there is a spectrum of potentially curative treatments."

While CT-based 3-dimensional conformal radiation therapy was in common use by 1995 , more novel treatment delivery methods that could better facilitate oligometastasisdirected treatment were not widely used in 1995. It was not until after the turn of the millennium that they were more widely adopted. Those advanced technologies include: (I) intensity-modulated radiotherapy (IMRT) which utilizes inverse planned computer algorithms to more conformally deliver the prescribed dose to the target while more readily sparing nearby normal tissues; (II) image-guided radiotherapy (IGRT) in which daily 
imaging that is incorporated into the treatment delivery system is used to more accurately position the patient and target the radiation, allowing for smaller margins for setup uncertainty; and (III) stereotactic body radiation therapy (SBRT) which utilizes technologies (including IGRT) that facilitate greater positioning accuracy, motion management (i.e., reducing or accounting for respiratory motion), and optimal patient immobilization. SBRT allows the delivery of very high fractional doses (i.e., dose per treatment), with a very steep dose gradient (meaning higher doses in the center of the target and lower doses in the periphery of the target) that facilitate better normal tissue sparing. The shorter treatment course of SBRT (one to a few treatments $v s$. several weeks with conventional radiotherapy) is also well-suited for patients with metastatic disease. Recognizing the limitations of the technology in 1995, and with some foresight into the development of IMRT and IGRT, Hellman and Weichselbaum noted that for metastasisdirected radiotherapy there was a need for an "... increase in the tumor dose and a reduction in normal tissue toxicity by restricting as much as possible, the radiation to the accurately imaged tumor while avoiding critical normal tissues". As with the Peters, Milas and Fletcher paper from a decade prior, Hellman and Weichselbaum's paper emphasized the need to minimize toxicity from radiotherapy when aggressively treating patients with metastatic disease.

\section{Treatment of oligometastatic disease with radiotherapy}

Historically, lung (8) and liver (9) oligometastatic disease had mostly been managed with surgical resection, with improved survival being observed in selected cancer types. Retrospective series have shown improved overall survival in patients undergoing lung resection for pulmonary metastases from high grade sarcoma $(10,11)$. Similarly, in colon cancer with limited liver metastasis, resection has been shown to improve survival $(12,13)$. Metastasectomy for renal carcinoma (14), most commonly for lung metastases (15), has been performed for decades. Data from these clinical scenarios support the principle of such an aggressive approach in oligometastatic disease. However, lung or liver metastatectomies are major invasive procedures, and only the most surgically fit patients are eligible. Over the next decades, with the advent of IMRT, IGRT and SBRT, many single- and multi-institutional single-arm prospective and retrospective studies were performed to study the impact of metastasis-directed ablative radiotherapy on outcomes among patients with oligometastases. Several of the papers in this special issue will review many of these studies.

SBRT provides a non-invasive means to ablate oligometastases in various organ sites, even in relatively less surgically fit patients. A recent review article highlighted that favorable prognostic factors among patients treated for oligometastases include younger age, greater performance status, more indolent disease course (i.e., metachronous vs. synchronous metastases, longer duration of time between primary cancer and metastatic diagnosis) and lower disease burden (fewer number of metastatic sites and organs involved) (16); certain caner types, such as breast or prostate cancer, are also associated with better outcomes. The main criticism of those studies published from the mid-2000s to 2010s was that without randomizing patients, it was unclear if patients fared better than expected simply because their disease was biologically different-with less aggressive growth and spread-and not impacted by the metastasisdirected treatment. In other words, the selection of patients with favorable prognosis (by virtue of having limited extent of metastatic disease) could account for their improved outcomes relative to all patients with more diffuse or aggressive Stage IV cancer of the same type. Furthermore, an immortal time bias can potentially be introduced leading to artificially more favorable outcomes associated with SBRT $(17,18)$.

In 2012, a multi-institutional randomized Phase II study, with 10 institutions in Canada, Netherlands, UK, and Australia, started enrolling patients with 1-5 oligometastases from any primary cancer type, with metastases to any organ/tissue. Patients were randomized (2:1 in favor of SBRT) to standard of care therapy with or without metastasis-directed SBRT (19). The studyStereotactic Ablative Radiotherapy for the Comprehensive Treatment of Oligometastases (SABR-COMET), published in 2018, ultimately enrolled 99 patients. The median progression-free survival was significantly longer (12 vs. 6 months, $\mathrm{P}=0.001)$ in patients randomized to receive SBRT; the difference in median overall survival (41 vs. 28 months, $\mathrm{P}=0.09$ ) met the study endpoint of $\mathrm{P}<0.20$, which suggested that a randomized Phase III study was warranted. Randomized Phase II studies in non-small cell lung cancer (NSCLC) (20-22) and prostate cancer $(23,24)$ similarly showed improvements in progressionfree survival (with the definition of progression being androgen-deprivation free survival for prostate cancer). While these studies are discussed in more detail in the papers from this special issue that are specific to NSCLC 
and prostate cancer, it is worth noting that as of 2020 , multiple randomized Phase II studies have demonstrated benefits in prolonging time to progression, and they have preliminarily suggested possible benefits in overall survival. Several randomized Phase III studies are accruing across the globe, including those for specific types of cancer (i.e., breast, prostate or lung cancer). These are also summarized in the papers included in this special issue.

Importantly, overall survival is a critical outcome measure, but it must be accompanied by toxicity and quality of life outcomes to best discern any potential clinical benefit of metastasis-directed radiotherapy. In the aforementioned SABR-COMET study, the authors noted (in a separate paper) small declines in quality of life measures among the entire cohort, with no apparent added benefit or detriment from metastasis-directed radiotherapy (25). Larger Phase III studies will be able to better address quality of life outcomes, though in general, improved cancer control and longer survival, in the absence of severe toxicity would be anticipated to be associated with better quality of life. This harkens back to the papers by Peters, Milas and Fletcher and Hellman and Weichselbaum, in which sparing of normal tissues from radiotherapy was emphasized. Patients with metastatic disease, for whom even if there is a subset that have curable disease, overall represent a group with relatively poor prognosis. Therefore, it is imperative to minimize toxicity. The novel technologies in radiotherapy developed over the past 2 decades have facilitated this.

\section{Identifying patients with 'true' oligometastatic disease}

In the era of precision medicine, the ultimate goal is to identify a priori patient-related and tumor-related factors that would allow clinicians to identify which treatments are best suited to each individual patient. This is particularly true for the treatment of oligometastases, as patients with metastatic disease can (at one extreme) experience rapid disease progression, and (at the other extreme) develop no additional sites of cancer. As with any cancer therapy, some patients will derive a benefit from a specific treatment, while others will not. Among those who received a treatment, the survival and cancer control outcomes may be directly attributable to the treatment in some, while some may have done just as well (or just as poorly) without treatment. Better predicting how the treatment for oligometastases would impact outcomes in a given patient would be a major step in catering the treatment to that patient.
The optimal selection of patients who would most benefit from metastasis-directed therapy is poorly understood. More work is needed in integrating molecular and genetic factors to better delineate where along the spectrum of metastatic disease a patient's cancer lies (26). Hypothesisgenerating studies from the University of Chicago analyzed specific microRNAs from patients who-for oligometastases from any primary site-underwent lung resection (27) or SBRT (to any site) $(28,29)$. Despite the various cancer histologies, microRNAs were able to differentiate oligometastatic vs. polymetastatic (i.e., those who developed widespread metastases) phenotypes; additionally, select microRNAs were able to convert stable oligometastases to polymetastatic progression in xenograft model. In an exploratory analysis of 17 patients, 3 microRNAs predicted survival (30). In an effort to develop a model for classification of oligometastases, three subtypes-with favorable (and few metastatic recurrences), intermediate and unfavorable survival-were derived from integrative molecular analyses (including immune activation markers) from 134 patients who underwent hepatic resection of limited liver metastases from colorectal cancer (31). The Moffitt Cancer center has developed a radiation sensitivity index, based upon gene expression assays, to predict for relative radiation sensitivity or resistance of oligometastases $(32,33)$. Despite promising preliminary findings from these studies, these molecular markers and bioassays have not yet been validated in larger studies.

Immunologic and/or inflammatory markers may also predict outcomes (34-36). With the advent of effective immunotherapy agents for cancer (particularly checkpoint inhibitors), there has been renewed interest in combining SBRT with immunotherapy to augment the effectiveness of immunotherapy. SBRT may accomplish this by altering tumor stroma (37), promoting the antigen presentation of target cancer cells, enhancing $\mathrm{T}$ cell infiltration, and other mechanisms (38-42). Checkpoint inhibitors may also counteract potential immuno-inhibitory effects of radiotherapy (39). In a recent study, a high number of regulatory $\mathrm{T}$ cells present before SBRT for oligometastases predicted overall and progression-free survival (43). More work is needed to fully understand the impact of SBRT on the immune response and how specific agents can be optimally used to maximize the immune response.

\section{Conclusions}

The clinical state of oligometastases represents, in some 
patients, a more indolent metastatic disease process for which metastasis-directed therapy can improve cancer control and survival outcomes. The notion that some patients might benefit from metastasis-directed therapy dates back decades. Hellman and Weichselbaum are credited with coining the term oligometastases and refining the oligometastases hypothesis in 1995 . However, it was not until the turn of the millennium that technologies were developed and refined to allow for the safe delivery of tumoricidal, short-course SBRT. The advent of these technologies led to a burgeoning of studies showing efficacy and tolerability of SBRT for oligometastatic disease. Promising results from randomized Phase II trials have led to ongoing Phase III studies. The next era of investigation will focus on means to use precision medicine to better identify patients who are apt to benefit from metastasisdirected therapy, as well as to augment the efficacy of systemic therapy (particularly immunotherapy) with SBRT. Most recently, European Society for Radiotherapy and Oncology (ESTRO) and European Organisation for Research and Treatment of Cancer (EORTC) recently published consensus recommendations for characteristics and classification of oligometastasis (44), which will be prospectively evaluated by the OligoCare study.

\section{Acknowledgments}

Funding: None.

\section{Footnote}

Provenance and Peer Review: This article was commissioned by the editorial office, Annals of Palliative Medicine for the series “Oligometastasis-Fallacy or Real Deal?". The article has undergone external peer review.

Conflicts of Interest: All authors have completed the ICMJE uniform disclosure form (available at http:// dx.doi.org/10.21037/apm.2020.03.31). The series “Oligometastasis-Fallacy or Real Deal?" was commissioned by the editorial office without any funding or sponsorship. All authors served as the unpaid Guest Editors of the series. MT Milano reports royalties from Wolters Kluwer and honorarium from Galera Therapeutics; T Biswas reports: honorarium from Galera Therapeutics; CB Simone II serves as the Editor-in-Chief of Annals of Palliative Medicine and reports honorarium from Varian Medical Systems; SS Lo serves as an unpaid Associate Editor-in-Chief of Annals of Palliative Medicine from Dec 2019 to Nov 2021 and reports: research support from Elekta $\mathrm{AB}$ for Gamma Knife ICON Expert Group. The authors have no other conflicts of interest to declare.

Ethical Statement: The authors are accountable for all aspects of the work in ensuring that questions related to the accuracy or integrity of any part of the work are appropriately investigated and resolved.

Open Access Statement: This is an Open Access article distributed in accordance with the Creative Commons Attribution-NonCommercial-NoDerivs 4.0 International License (CC BY-NC-ND 4.0), which permits the noncommercial replication and distribution of the article with the strict proviso that no changes or edits are made and the original work is properly cited (including links to both the formal publication through the relevant DOI and the license). See: https://creativecommons.org/licenses/by-nc-nd/4.0/.

\section{References}

1. Correa RJ, Salama JK, Milano MT, et al. Stereotactic body radiotherapy for oligometastasis: Opportunities for biology to guide clinical management. Cancer J 2016;22:247-56.

2. Rubin P Green J. Solitary metastases. Springfield, IL: C. C. Thomas, 1968.

3. Rosenberg SA. Surgical treatment of metastatic cancer. Philadelphia, PA: Lippincott Williams \& Wilkins, 1987.

4. Peters LJ, Milas L, Fletcher GH. The role of radiation therapy in the curative treatment of metastatic disease. Symp Fundam Cancer Res 1983;36:411-420.

5. Rubin P. Comment: Are metastases curable? JAMA 1968;204:612-3.

6. Hellman S. Karnofsky memorial lecture. Natural history of small breast cancers. J Clin Oncol 1994;12:2229-34.

7. Hellman S Weichselbaum RR. Oligometastases. J Clin Oncol 1995;13:8-10.

8. Pastorino U, Buyse M, Friedel G, et al. Long-term results of lung metastasectomy: Prognostic analyses based on 5206 cases. J Thorac Cardiovasc Surg 1997;113:37-49.

9. Kuvshinoff B Fong Y. Surgical therapy of liver metastases. Semin Oncol 2007;34:177-85.

10. Ueda T, Uchida A, Kodama K, et al. Aggressive pulmonary metastasectomy for soft tissue sarcomas. Cancer 1993;72:1919-25.

11. Billingsley KG, Burt ME, Jara E, et al. Pulmonary metastases from soft tissue sarcoma: Analysis of patterns 
of diseases and postmetastasis survival. Ann Surg 1999;229:602-10; discussion 610-2.

12. Kanas GP, Taylor A, Primrose JN, et al. Survival after liver resection in metastatic colorectal cancer: Review and meta-analysis of prognostic factors. Clin Epidemiol 2012;4:283-301.

13. Brown RE, Bower MR Martin RC. Hepatic resection for colorectal liver metastases. Surg Clin North Am 2010;90:839-52.

14. Ouzaid I, Capitanio U, Staehler M, et al. Surgical metastasectomy in renal cell carcinoma: A systematic review. Eur Urol Oncol 2019;2:141-9.

15. Zhao Y, Li J, Li C, et al. Prognostic factors for overall survival after lung metastasectomy in renal cell cancer patients: A systematic review and meta-analysis. Int J Surg 2017;41:70-7.

16. Palma DA, Louie AV Rodrigues GB. New strategies in stereotactic radiotherapy for oligometastases. Clin Cancer Res 2015;21:5198-5204.

17. Levesque LE, Hanley JA, Kezouh A, et al. Problem of immortal time bias in cohort studies: Example using statins for preventing progression of diabetes. BMJ 2010;340:b5087.

18. Palma DA, Salama JK, Lo SS, et al. The oligometastatic state - separating truth from wishful thinking. Nat Rev Clin Oncol 2014;11:549-57.

19. Palma DA, Olson R, Harrow S, et al. Stereotactic ablative radiotherapy versus standard of care palliative treatment in patients with oligometastatic cancers (SABRCOMET): A randomised, phase 2, open-label trial. Lancet 2019;393:2051-8.

20. Gomez DR, Blumenschein GR Jr, Lee JJ, et al. Local consolidative therapy versus maintenance therapy or observation for patients with oligometastatic non-smallcell lung cancer without progression after first-line systemic therapy: A multicentre, randomised, controlled, phase 2 study. Lancet Oncol 2016;17:1672-82.

21. Gomez DR, Tang C, Zhang J, et al. Local consolidative therapy vs. Maintenance therapy or observation for patients with oligometastatic non-small-cell lung cancer: Long-term results of a multi-institutional, phase ii, randomized study. J Clin Oncol 2019;37:1558-65.

22. Iyengar P, Wardak Z, Gerber DE, et al. Consolidative radiotherapy for limited metastatic non-small-cell lung cancer: A phase 2 randomized clinical trial. JAMA Oncol 2018;4:e173501.

23. Ost P, Reynders D, Decaestecker K, et al. Surveillance or metastasis-directed therapy for oligometastatic prostate cancer recurrence: A prospective, randomized, multicenter phase ii trial. J Clin Oncol 2018;36:446-53.

24. Phillips R, Shi WY, Deek M, et al. Outcomes of observation vs stereotactic ablative radiation for oligometastatic prostate cancer: The ORIOLE phase 2 randomized clinical trial. JAMA Oncol 2020;6:650-9.

25. Olson R, Senan S, Harrow S, et al. Quality of life outcomes after stereotactic ablative radiation therapy (SABR) versus standard of care treatments in the oligometastatic setting: A secondary analysis of the SABR-COMET randomized trial. Int J Radiat Oncol Biol Phys 2019;105:943-7.

26. Pitroda SP Weichselbaum RR. Integrated molecular and clinical staging defines the spectrum of metastatic cancer. Nat Rev Clin Oncol 2019;16:581-8.

27. Lussier YA, Khodarev NN, Regan K, et al. Oligo- and polymetastatic progression in lung metastasis(es) patients is associated with specific microRNAs. PLoS One 2012;7:e50141.

28. Lussier YA, Xing HR, Salama JK, et al. MicroRNAs expression characterizes oligometastasis(es). PLoS One 2011;6:e28650.

29. Uppal A, Ferguson MK, Posner MC, et al. Towards a molecular basis of oligometastatic disease: Potential role of micro-RNAs. Clin Exp Metastasis 2014;31:735-48.

30. Wong AC, Watson SP, Pitroda SP, et al. Clinical and molecular markers of long-term survival after oligometastasis-directed stereotactic body radiotherapy (SBRT). Cancer 2016;122:2242-50.

31. Pitroda SP, Khodarev NN, Huang L, et al. Integrated molecular subtyping defines a curable oligometastatic state in colorectal liver metastasis. Nat Commun 2018;9:1793.

32. Ahmed KA, Fulp WJ, Berglund AE, et al. Differences between colon cancer primaries and metastases using a molecular assay for tumor radiation sensitivity suggest implications for potential oligometastatic SBRT patient selection. Int J Radiat Oncol Biol Phys 2015;92:837-42.

33. Ahmed KA, Berglund AE, Welsh EA, et al. Differences in the radiosensitivity index (RSI) between lung metastases based upon primary histology. Int J Radiat Oncol Biol Phys 2016;96:S68.

34. Ishikawa H, Metcalfe SK, Milano MT, et al. The impact of gm-csf up-regulation by SBRT on overall survival of metastatic breast cancer. Int J Radiat Oncol Biol Phys 2011;75:S539.

35. Metcalfe SK, Ishikawa H, Milano MT, et al. Gm-CSF and IFN-gamma up-regulation with stereotactic body radiation therapy for oligometastatic breast cancer correlates with improved overall survival. American Radium Society 92nd 
Annual Meeting, 2010.

36. Muraro E, Furlan C, Avanzo M, et al. Local high-dose radiotherapy induces systemic immunomodulating effects of potential therapeutic relevance in oligometastatic breast cancer. Front Immunol 2017;8:1476.

37. Menon H, Ramapriyan R, Cushman TR, et al. Role of radiation therapy in modulation of the tumor stroma and microenvironment. Front Immunol 2019;10:193.

38. Cushman TR, Gomez D, Kumar R, et al. Combining radiation plus immunotherapy to improve systemic immune response. J Thorac Dis 2018;10:S468-79.

39. Pitroda SP, Chmura SJ, Weichselbaum RR. Integration of radiotherapy and immunotherapy for treatment of oligometastases. Lancet Oncol 2019;20:e434-42.

40. Weichselbaum RR, Liang H, Deng L, et al. Radiotherapy and immunotherapy: A beneficial liaison? Nat Rev Clin Oncol 2017;14:365-79.

Cite this article as: Milano MT, Biswas T, Simone CB 2nd, Lo SS. Oligometastases: history of a hypothesis. Ann Palliat Med 2021;10(5):5923-5930. doi: 10.21037/apm.2020.03.31
41. Weichselbaum RR. The 46th David A. Karnofsky Memorial Award Lecture: Oligometastasis-From Conception to Treatment. J Clin Oncol 2018;36:3240-50.

42. Onderdonk BE Chmura SJ. The yin and yang of cytoreductive SBRT in oligometastases and beyond. Front Oncol 2019;9:706.

43. Liu C, Sun B, Hu X, et al. Stereotactic ablative radiation therapy for pulmonary recurrence-based oligometastatic non-small cell lung cancer: Survival and prognostic value of regulatory t cells. Int J Radiat Oncol Biol Phys 2019;105:1055-64.

44. Guckenberger M, Lievens Y, Bouma AB, et al. Characterisation and classification of oligometastatic disease: A european society for radiotherapy and oncology and european organisation for research and treatment of cancer consensus recommendation. Lancet Oncol 2020;21:e18-28. 